\title{
广西扶绥白头叶猴对栖息地的选择与利用
}

\author{
何雪李 ${ }^{1,2}$, 陆施毅 ${ }^{1,2}$, 黄中豪 ${ }^{1,2}$, 李友邦 ${ }^{1,2, *}$ \\ 1 珍稀濒危动植物生态与环境保护教育部重点实验室,广西师范大学,桂林 541006 \\ 2 广西珍稀濒危动物生态学重点实验室,广西师范大学,桂林 541006
}

摘要: 为了解白头叶猴 (Trachypithecus leucocephalus) 的栖息地利用规律及其影响因素,2016 年 2 月至 2017 年 1 月,采用瞬时扫 描取样法对广西崇左白头叶猴国家级自然保护区一群白头叶猴的栖息地利用进行了研究。结果表明,白头叶猴对山体不同部 位的利用存在显著性差异 $\left(\chi^{2}=39.467, d f=3, P<0.001\right)$, 其中, 对崖壁 $(56.75 \pm 9.55) \%$ 的利用比例最大, 其次是对山坡( $39.42 \pm$ $10.93) \%$ 和山顶 $(2.98 \pm 2.54) \%$ 的利用,而对山脚 $(0.84 \pm 1.47) \%$ 的利用频率最低。白头叶猴对不同微生境类型的利用存在差异 $\left(\chi^{2}=27.709, d f=3, P<0.001\right)$, 其中对乔木 $(49.37 \pm 12.31) \%$ 的利用比例最大, 其次是裸岩 $(24.05 \pm 13.61) \%$, 随后依次为藤本 $(15.48 \pm 8.01) \%$ 和灌木 $(10.87 \pm 5.45) \%$ 。白头叶猴主要在山坡上受食, 利用崖壁移动、休息,进行社会活动; 主要利用裸岩进行 社会活动, 受食、移动、休息主要发生在乔木上。从整体来看, 白头叶猴在雨季对乔木的利用频率显著大于旱季 $(Z=-2.680, n=$ $12, P=0.007)$; 雨季在山坡受食频率显著大于旱季 $(Z=-2.517, n=12, P=0.012)$, 而在崖壁受食频率刚好相反 $(Z=-2.842, n=$ $12, P=0.004)$; 白头叶猴雨季在乔木休息的频率显著大于旱季 $(Z=-2.355, n=12, P=0.019)$ 。白头叶猴对栖息地的利用受到温 度的影响。白头叶猴对乔木的总体利用频率随着平均温度的升高而增加 $(r=0.664, n=12, P=0.018)$; 受食时, 对崖壁、裸岩的 利用频率均与平均温度成负相关关系 (崖壁: $r=-0.685, n=12, P=0.014$; 裸岩: $r=-0.600, n=12, P=0.039$ ); 休息时,对乔木的利 用频率与平均温度呈正相关关系 $(r=0.650, n=12, P=0.022)$ 。不同季节,白头叶猴对栖息地的利用方式不同。白头叶猴的栖 息地利用模式可能是在受食利益和捕食风险之间作出的权衡,并受到环境温度的影响。

关键词: 白头叶猴;栖息地利用; 喀斯特石山;行为温度调节

\section{Habitat selection and use of a group of white-headed langurs (Trachypithecus leucocephalus) in Fusui, Guangxi, China}

HE Xueli ${ }^{1,2}$, LU Shiyi ${ }^{1,2}$, HUANG Zhonghao ${ }^{1,2}$, LI Youbang ${ }^{1,2, *}$

1 Key Laboratory of Ecology of Rare and Endangered Species and Environmental Protection ( Guangxi Normal University), Ministry of Education, Guilin 541006, China

2 Guangxi Key Laboratory of Rare and Endangered Animal Ecology, Guangxi Normal University, Guilin 541006, China

\begin{abstract}
In order to understand the habitat utilization rule of the white-headed langur and its influencing factors, a group of white-headed langurs ( Trachypithecus leucocephalus) was investigated from February 2016 to January 2017 in the Chongzuo White-headed Langur National Nature Reserve, Guangxi, China by instantaneous scanning sampling method. The results indicated that significant difference in the utilization of different limestone hill parts of white-headed langur was found $\left(\chi^{2}=39.467, d f=3, P<0.001\right)$. The cliff was used most frequently $(56.75 \pm 9.55) \%$, followed by the slope $(39.42 \pm 10.93) \%$, the hill-top $(2.98 \pm 2.54) \%$, as well as the valley $(0.84 \pm 1.47) \%$. There were significant differences in the utilization of different microhabitat types of langurs $\left(\chi^{2}=27.709, d f=3, P<0.001\right)$. Tree as the preferred
\end{abstract}

基金项目:国家自然科学基金项目(31960104, 31960106)

收稿日期: 2020-08-26; 网络出版日期: 2021-07-05

* 通讯作者 Corresponding author.E-mail: lyb_2001@126.com 
microhabitat, and its percentage $(49.37 \pm 12.31) \%$ was higher than bare rock $(24.05 \pm 13.61) \%$, liana $(15.48 \pm 8.01) \%$, and bush $(10.87 \pm 5.45) \%$. The langurs mainly fed on slope, and other activities, such as moving, resting and social activity were on cliff. Besides, social activity was the only behavior occurred most often in bare rock, while other behaviors occurred most often in trees. There were seasonal differences in limestone hill parts and microhabitat. In overall activities, langurs used trees significantly more in the rainy season than in the dry season $(Z=-2.680, n=12, P=0.007)$. When feeding, langurs used slope significantly more in the rainy season than in the dry season $(Z=-2.517, n=12, P=0.012)$, while the use of cliff was opposite $(Z=-2.842, n=12, P=0.004)$. When resting, in the rainy season the langurs used trees significantly more than in the dry season $(Z=-2.355, n=12, P=0.019)$. Habitat use of white-headed langurs was also influenced by temperature. The utilization frequency of overall activities of the trees increased with the average temperature $(r=0.664, n=12, P=0.018)$. Besides, the utilization frequency of resting of the trees increased with the average temperature $(r=0.650, n=12, P=0.022)$. In contrast, the utilization frequency of cliff and bare rock in feeding was both negatively correlated with the average temperature (cliff : $r=-0.685, n=12, P=0.014$; bare rock: $r=-0.600$, $n=12, P=0.039)$. In different seasons, white-headed langurs used its habitat in different ways. Thus, the habitat utilization pattern of white-headed langurs may be a trade-off between feeding benefits and predation risk, and is also influenced by ambient temperature.

Key Words: Trachypithecus leucocephalus; habitat use; limestone forest; behavioral thermoregulation

栖息地是动物生存的空间,决定着食物资源、隐蔽条件等, 最终影响着动物的生存和繁衍 ${ }^{[1]}$ 。影响灵长 类栖息地选择与利用的因素众多,包括人为干扰 ${ }^{[2-3]}$ 、气候条件 ${ }^{[4-7]}$ 、受食利益与捕食风险 ${ }^{[4-5,8-10]}$ 等。以受食 利益和捕食风险为例, 石山地区的熊猴 (Macaca assamensis) 和狋猴 (M. mulatta) 均偏向选择喜食食物丰富的 山体部位 ${ }^{[11]}$; 同域分布的黑叶猴 (Trachypithecus francoisi) 在受食利益与捕食风险之间进行权衡, 当山脚部位 高质量食物更丰富时,猴群会增加对该山体部位的利用; 当黑叶猴在山顶找到花、成熟叶等替代食物时,则减 少对山脚的利用,更倾向于在低风险的山顶受食 ${ }^{[5]}$ 。

栖息地的气候因素也会影响灵长类对栖息地的利用。一方面,降雨量等气候因子会影响森林中的嫩叶、 花、果实等数量 ${ }^{[12-13]}$, 可能对灵长类的栖息地选择产生间接影响; 另一方面, 气候因素, 特别是温度, 对灵长类 栖息地利用产生直接影响 ${ }^{[14]}$ 。当环境温度低时, 灵长类会选择适合的栖息地以减少身体热量的丧失或者获 得额外的热量,如倾向于利用阳光充足的坡向 (太行山猕猴 ${ }^{[15] \text { 、黑叶猴 }}{ }^{[16]}$ ) 等。在高温环境中灵长类则采用 相反的策略。灵长类倾向于利用阴凉的地方以躲避高温, 降低体表温度, 如卷尾猴 (Cebus capucinus) ${ }^{[17]}$ 、黑吼 猴 (Alouatta caraya $)^{[14]}$ 、青腹绿猴 (Chlorocebus pygerythrus $)^{[7]}$ 和黑叶猴 ${ }^{[6]}$ 等。

白头叶猴 (Trachypithecus leucocephalus) 是中国特有的灵长类, 仅分布在广西南部一个面积约 $200 \mathrm{~km}^{2}$ 的狭 窄三角地带 ${ }^{[18]}$, 在 IUCN 物种红色名录中被列为 “极危” 级别 ${ }^{[19]}$ 。白头叶猴的栖息环境为喀斯特石山, 悬崖 峭壁密布, 晴天太阳升起后, 裸露岩石表面温度迅速上升, 并在中午达到最高值, 而树荫下的温度明显低于裸 岩的温度 ${ }^{[18]}$ 。石山自上而下可划分为山顶、崖壁、山坡和山脚 ${ }^{[16,18,20]}$, 石山之间的山弄平地被当地居民用于 种植农作物等 ${ }^{[21]}$,栖息环境被人类活动高度分割, 栖息地破碎化十分严重 ${ }^{[22]}$ 。栖息地丧失与破碎化是造成 野生动物濒危和灭绝的主要原因 ${ }^{[23]}$, 对生活在极易破碎化森林栖息地的白头叶猴来说尤其如此。因此,开展 白头叶猴生境选择的研究可了解其对栖息地的反应,为理解其对栖息地破碎化的适应策略有重要意义。前期 研究发现, 白头叶猴会选择较为安全且食物相对丰富的山体中部作为主要的受食场所,而较少利用人为活动 较为频繁的山体下部 ${ }^{[3]}$, 只有大猴群因食物资源需求压力会增加对山体下部的利用 ${ }^{[24]}$ 。然而, 上述研究并不 能完全反映出白头叶猴对石山生境的利用规律。例如,白头叶猴对微生境的利用方式如何? 不同行为类型、 不同季节下是否采取相同的栖息地利用对策? 温度对栖息地利用有何影响? 研究系统地收集了广西崇左白 头叶猴国家级自然保护区喀斯特石山生境中一群白头叶猴的生境利用数据, 以期深人了解该濒危物种对栖息 
地破碎化的适应策略。栖息地利用研究表明,灵长类会根据不同的行为和季节,以不同的方式利用栖息地。 因此,提出以下假设:（1)基于对捕食风险和受食利益的权衡,白头叶猴总体上倾向在崖壁活动,较少在山顶 和山脚活动; 受食时倾向在山坡活动,这种倾向在食物丰富的雨季可能更为突出;(2) 基于行为热调节策略, 白头叶猴在寒冷的旱季倾向于利用崖壁和裸岩,且温度越低这种倾向越明显; 而炎热的雨季倾向利用乔木,温 度越高这种倾向越明显。

\section{1 研究方法}

1.1 研究地点与研究对象

2016 年 2 月至 2017 年 1 月, 在广西崇左白头叶猴 国家级自然保护区 ( 以下简称“白头叶猴保护区”) 九重 山片区开展研究。白头叶猴保护区位于广西西南部, 属 于亚热带季风气候, 具有明显的雨季和旱季, 雨季为 5 月一 9 月, 旱季为 10 月至次年 4 月 ${ }^{[25]}$ 。研究期间, 借助 HOBO 自动温度/湿度记录仪记录环境的温度。结果表 明年平均温度为 $29.3^{\circ} \mathrm{C}$, 月平均温度在 $17.5^{\circ} \mathrm{C}$ ( 1 月 ) 至 $34.8^{\circ} \mathrm{C}$ ( 7 月) 之间变化 (图 1)。

研究对象为保护区内 1 群白头叶猴,由 1 只成年雄 猴、4 只成年雌猴、6 只亚成年猴、3 只青年猴、1 只婴幼 猴, 共 15 个个体组成。

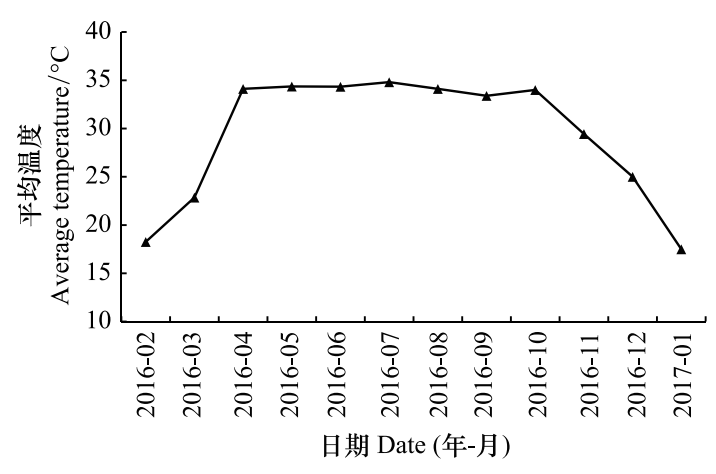

图 1 广西崇左白头叶猴保护区的平均温度

Fig.1 Monthly average temperature in the study site during the study period

\section{2 数据收集}

研究期间,采用瞬时扫描取样法 ${ }^{[26]}$ 收集白头叶猴的行为、山体部位和微生境利用的数据。以 $15 \mathrm{~min}$ 为 一个扫描单元进行取样,每次扫描时间持续 $5 \mathrm{~min}$, 间隔 $10 \mathrm{~min}$ 后开始下一次扫描取样。从猴群的最左侧开 始扫描, 依次观察所见个体。由于婴幼猴通常是由雌猴携带的, 因此记录下除婴幼猴外每个个体的行为类型、 所处的山体部位和微生境类型。山体部位包括山顶、崖壁、山坡和山脚。微生境类型包括乔木、灌木、藤本、草 本和裸岩。藤本和草本较容易划分, 乔木和灌木的划分参照文献 ${ }^{[27]}$ 。行为类型的定义参照文献 ${ }^{[28-30]}$, 记录为 受食、移动、休息、社会活动和其他行为。在完整观察日, 从清晨猴群离开夜宿地开始取样, 直至傍晚时, 猴群 全部回到夜宿地结束当天的取样。观察期间,共收集到 50 个完整观察日的数据,扫描次数 2040 次 (表 1)。

表 1 白头叶猴的取样数量

Table 1 Number of scans of white-headed langur

\begin{tabular}{lrrrrrrrrrrrrrr}
\hline & \multicolumn{10}{c}{ 日期 Date(年-月) } \\
\cline { 2 - 14 } & $2010-02$ & $2010-03$ & $2010-04$ & $2010-05$ & $2010-06$ & $2010-07$ & $2010-08$ & $2010-09$ & $2010-10$ & $2010-11$ & $2010-12$ & $2010-01$ & 总计 \\
\hline 完整观察日 Full observation day & 5 & 5 & 5 & 4 & 4 & 4 & 4 & 4 & 2 * & 5 & 4 & 4 & 50 \\
扫描次数 Scan times & 201 & 212 & 210 & 172 & 192 & 184 & 178 & 158 & 80 & 186 & 141 & 126 & 2040 \\
\hline
\end{tabular}

$* 10$ 月因降雨淹没观察地点, 且淹没时间较长, 仅收集到 2 个完整观察日的数据

\section{3 数据处理与分析}

参照文献 ${ }^{[5,31]}$ 将每次扫描取样中大部分个体的行为类型定义为该时段猴群的主要行为类型, 将大部分个 体所处的山体部位和微生境类型代表该时段猴群所利用的山体部位和微生境类型, 以分析栖息地的使用情 况。以每次扫描取样作为一个独立样本, 将猴群每日对不同生境 (山体部位、微生境类型) 的利用次数除以一 天记录的总次数,计算出每日对不同生境类型的利用比例, 再对每个完整观察日的利用比例进行平均得到每 月的利用比例, 然后对每月的值进一步平均得到不同季节和全年的利用比例。该方法同样用于不同山体下对 不同微生境类型的利用以及各行为类型下对山体不同部位、微生境类型的利用。 
统计分析时, 先对所有百分比数据进行 logit 转换, 非百分比数据进行 $\log (X)$ 转换, 然后用 KolmogorovSmirnov 检验数据分布的正态性。结果表明,部分数据非正态分布 $(P<0.05)$ 。因此,采用 Kruskal-Wallis 检验 来比较多个变量的差异, 采用 Mann-Whitney U 检验来比较两个独立样本间的差异, 采用 Spearman 检验温度和 栖息地利用的相关性。由于猴群对草本的利用频率仅为 $0.23 \%$,因此不对其作进一步分析。数据的处理和分 析在 Excel 2010 和 SPSS 25.0 软件上完成,显著性水平设为 0.05 。

\section{2 结果}

2.1 白头叶猴对山体不同部位和微生境的利用

白头叶猴对山体不同部位的利用存在差异 $\left(\chi^{2}=39.467, d f=3, P<0.001\right)$, 其中崖壁的利用比例最大 (56.75 \pm 9.55$) \%$, 其次是山坡 $(39.42 \pm 10.93) \%$ 和山顶 $(2.98 \pm 2.54) \%$, 山脚的利用频率最低, 仅为 $(0.84 \pm$ $1.47) \%$ 。白头叶猴对不同微生境类型的利用具有选择性 $\left(\chi^{2}=27.709, d f=3, P<0.001\right)$, 其中乔木的利用比例 最大 $(49.37 \pm 12.31) \%$, 后面依次是裸岩 $(24.05 \pm 13.61) \%$ 、藤本 $(15.48 \pm 8.01) \%$ 和灌木 $(10.87 \pm 5.45) \%$ 。白头 叶猴对乔木、灌木、藤本、裸岩的利用在山体不同部位下具有选择性 (乔木: $\chi^{2}=14.623, d f=3, P=0.002$; 灌木: $\chi^{2}=10.551, d f=3, P=0.014$; 藤本 : $\chi^{2}=41.170, d f=3, P<0.001$; 裸岩: $\left.\chi^{2}=30.330, d f=3, P<0.001\right)$ 。白头叶猴对 乔木的利用主要在山坡和山脚; 对灌木的利用主要在山脚; 对藤本的利用主要在崖壁和山坡; 对裸岩的利用主 要在山顶和崖壁(图 2)。

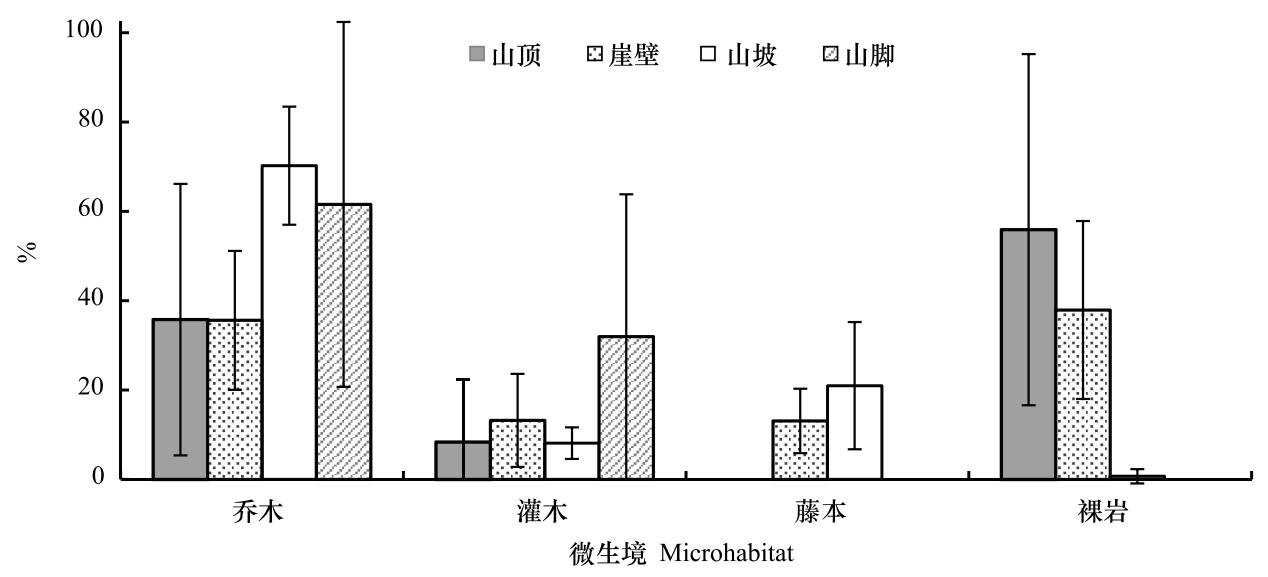

图 2 白头叶猴在不同山体下对各微生境的利用

Fig.2 Use of microhabitat in different limestone hill parts by white-headed langur

白头叶猴在各行为类型下对山体不同部位、微生境类型的利用表现出明显的差异性(表 2)。受食时利用 最多的山体部位是山坡,依次是崖壁、山顶、山脚 (图 3)。受食主要在乔木上,随后是藤本、灌木, 而对裸岩的 利用很少 (图 3)。移动、休息和社会活动时,对崖壁的利用比例最高, 依次是山坡、山顶、山脚(图 3)。移动、 休息时主要在乔木上, 依次是裸岩、藤本、灌木, 而社会活动时主要在裸岩上, 其次是乔木, 对灌木、藤本的利用 频率较低 (图 3)。

2.2 白头叶猴对山体不同部位和微生境利用的季节性变化

白头叶猴在雨季和旱季对山体部位的利用存在显著差异 (表 3)。受食时,在雨季对山坡的利用频率明显 高于旱季, 同时相应的减少对崖壁的利用频率 (表 3)。猴群在雨季和旱季对微生境的利用也存在显著差异 (表 4)。从总体来看, 猴群在雨季对乔木的利用频率明显高于旱季 (表 4)。休息时, 雨季对乔木的利用频率 明显高于旱季 (表 4 )。 
表 2 白头叶猴在不同行为类型下对山体部位、微生境利用的 Kruskal-Wallis 检验结果

Table 2 Results of Kruskal-Wallis test of behaviors across limestone hill parts and microhabitat among white-headed langur

\begin{tabular}{lccccc}
\hline & \multicolumn{4}{c}{ 行为 Behavior } \\
\cline { 3 - 6 } & $\chi^{2}$ & 受食 Feeding & 移动 Moving & 休息 Resting & 社会活动 Social activity \\
\hline 山体部位 Hill parts & 37.950 & 40.443 & 41.186 & 37.251 \\
& $d f$ & 3 & 3 & 3 & 3 \\
微生境 Microhabitat & $P$ & $<0.001$ & $<0.001$ & $<0.001$ & $<0.001$ \\
& $\chi^{2}$ & 36.295 & 30.554 & 3 & 33.351 \\
& $d f$ & 3 & 3 & $<0.001$ & 3 \\
& $P$ & $<0.001$ & $<0.001$ & & $<.001$ \\
\hline
\end{tabular}
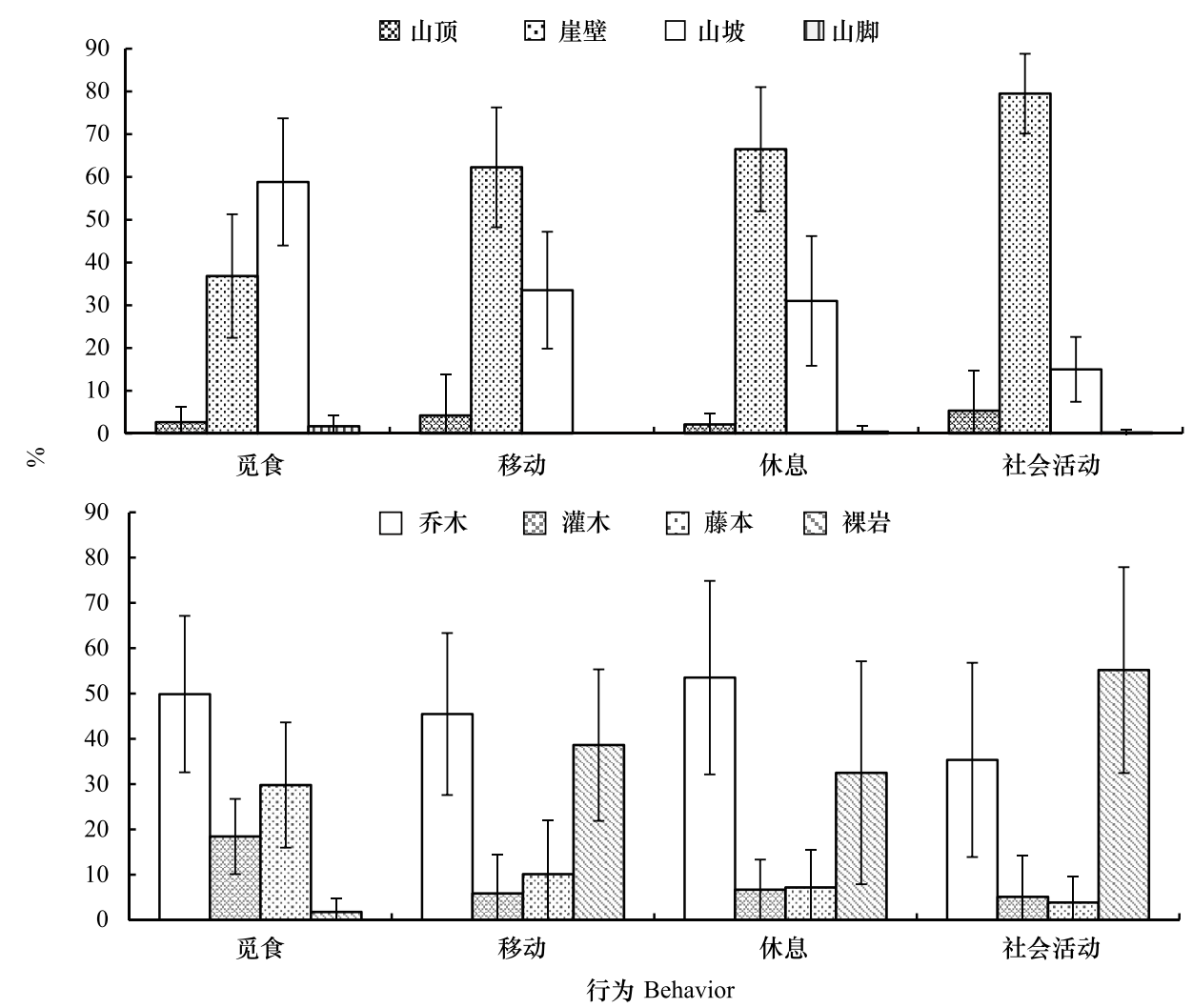

图 3 白头叶猴在不同行为类型下对山体部位、微生境的利用

Fig.3 Activities occurred in different limestone hill parts and microhabitat by white-headed langur

表 3 白头叶猴在雨季和旱季对山体部位的利用 $(n=12)$

Table 3 Usage of limestone hill parts by white-headed langur in rainy and dry seasons $(n=12)$

\begin{tabular}{cccccc}
\hline & & \multicolumn{4}{c}{ 山体部位 Hill part } \\
\cline { 3 - 6 } & & 山顶 Hill-top & 崖壁 Cliff & 山坡 Slope & 山脚 Valley \\
\hline 总体 Overall & 雨季/\% & $2.75 \pm 1.82$ & $50.67 \pm 11.03$ & $46.04 \pm 12.91$ & $0.54 \pm 0.55$ \\
& 旱季/\% & $3.15 \pm 3.08$ & $61.09 \pm 5.79$ & $34.70 \pm 6.75$ & $1.06 \pm 1.91$ \\
& $Z$ & -0.488 & -1.705 & -1.705 & -0.087 \\
愛食 Feeding & $P$ & 0.626 & 0.088 & 0.088 & 0.931 \\
& 雨季/\% & $3.98 \pm 5.42$ & $23.69 \pm 11.07$ & $70.96 \pm 15.00$ & $1.37 \pm 1.47$ \\
& 旱季/\% & $1.66 \pm 1.37$ & $46.21 \pm 7.39$ & $50.18 \pm 6.75$ & $1.94 \pm 3.01$ \\
& $Z$ & -0.083 & -2.842 & -2.517 & -0.260 \\
\hline
\end{tabular}




\begin{tabular}{|c|c|c|c|c|c|}
\hline \multicolumn{6}{|c|}{ 山体部位 Hill part } \\
\hline & & 山顶 Hill-top & 崖壁 Cliff & 山坡 Slope & 山脚 Valley \\
\hline \multirow[t]{4}{*}{ 移动 Moving } & 雨季/\% & $0.83 \pm 1.86$ & $65.42 \pm 8.01$ & $33.75 \pm 6.65$ & 0 \\
\hline & 旱季/\% & $6.61 \pm 12.17$ & $60.02 \pm 17.42$ & $33.37 \pm 17.69$ & 0 \\
\hline & $Z$ & -1.063 & -0.163 & -0.570 & 0 \\
\hline & $P$ & 0.288 & 0.871 & 0.568 & 1 \\
\hline \multirow[t]{4}{*}{ 休息 Resting } & 雨季/\% & $2.65 \pm 3.81$ & $62.14 \pm 19.40$ & $35.20 \pm 20.61$ & 0 \\
\hline & 旱季/\% & $1.72 \pm 1.78$ & $69.64 \pm 10.42$ & $27.98 \pm 10.63$ & $0.66 \pm 1.74$ \\
\hline & $Z$ & 0 & -1.056 & -0.568 & -0.845 \\
\hline & $P$ & 1 & 0.291 & 0.570 & 0.398 \\
\hline \multirow[t]{4}{*}{ 社会活动 Social activity } & 雨季/\% & $1.67 \pm 3.73$ & $81.50 \pm 8.53$ & $16.83 \pm 6.23$ & 0 \\
\hline & 旱季/\% & $7.92 \pm 11.62$ & $78.06 \pm 10.28$ & $13.67 \pm 8.63$ & $0.36 \pm 0.94$ \\
\hline & $Z$ & -1.177 & -0.568 & -0.731 & -0.845 \\
\hline & $P$ & 0.239 & 0.570 & 0.465 & 0.398 \\
\hline
\end{tabular}

表 4 白头叶猴在雨季和旱季对微生境的利用 $(n=12)$

Table 4 Usage of microhabitat by white-headed langur in rainy and dry seasons $(n=12)$

\begin{tabular}{|c|c|c|c|c|c|}
\hline & & \multicolumn{4}{|c|}{ 微生境 Microhabitat/\% } \\
\hline & & 乔木 Tree & 灌木 Bush & 藤本 Liana & 裸岩 Bare rock \\
\hline \multirow[t]{4}{*}{ 总体 Overall } & 雨季/\% & $61.10 \pm 4.60$ & $10.57 \pm 4.33$ & $12.71 \pm 3.04$ & $15.61 \pm 8.45$ \\
\hline & 旱季/\% & $40.99 \pm 8.18$ & $11.09 \pm 6.46$ & $17.45 \pm 10.03$ & $30.08 \pm 13.80$ \\
\hline & $Z$ & -2.680 & -0.081 & -1.056 & -1.705 \\
\hline & $P$ & 0.007 & 0.935 & 0.291 & 0.088 \\
\hline \multirow[t]{4}{*}{ 受食 Feeding } & 雨季/\% & $57.42 \pm 13.51$ & $18.05 \pm 6.35$ & $24.26 \pm 7.96$ & $0.26 \pm 0.59$ \\
\hline & 旱季/\% & $44.47 \pm 18.56$ & $18.69 \pm 9.97$ & $33.72 \pm 16.26$ & $2.83 \pm 3.47$ \\
\hline & $Z$ & -1.056 & -0.244 & -1.380 & -1.539 \\
\hline & $P$ & 0.291 & 0.808 & 0.167 & 0.124 \\
\hline \multirow[t]{4}{*}{ 移动 Moving } & 雨季/\% & $42.50 \pm 7.88$ & $4.17 \pm 5.89$ & $14.17 \pm 15.12$ & $39.17 \pm 20.17$ \\
\hline & 旱季/\% & $47.58 \pm 23.10$ & $7.02 \pm 10.36$ & $7.20 \pm 9.21$ & $38.19 \pm 15.55$ \\
\hline & $Z$ & -0.081 & -0.261 & -1.014 & -0.244 \\
\hline & $P$ & 0.935 & 0.794 & 0.310 & 0.807 \\
\hline \multirow[t]{4}{*}{ 休息 Resting } & 雨季/\% & $70.42 \pm 15.70$ & $5.91 \pm 4.02$ & $5.28 \pm 8.14$ & $18.39 \pm 15.66$ \\
\hline & 旱季/\% & $41.42 \pm 16.26$ & $7.19 \pm 8.37$ & $8.53 \pm 8.82$ & $42.56 \pm 25.74$ \\
\hline & $Z$ & -2.355 & -0.245 & -0.895 & -1.543 \\
\hline & $P$ & 0.019 & 0.806 & 0.371 & 0.123 \\
\hline \multirow[t]{4}{*}{ 社会活动 Social activity } & 雨季/\% & $41.88 \pm 27.19$ & $2.59 \pm 2.65$ & $3.75 \pm 8.39$ & $51.79 \pm 22.73$ \\
\hline & 旱季/\% & $30.67 \pm 17.04$ & $6.90 \pm 11.77$ & $3.88 \pm 3.76$ & $57.58 \pm 24.23$ \\
\hline & $Z$ & -0.731 & -0.087 & -0.815 & -0.568 \\
\hline & $P$ & 0.465 & 0.931 & 0.415 & 0.570 \\
\hline
\end{tabular}

2.3 温度对白头叶猴山体部位和微生境利用的影响

白头叶猴对山体部位和微生境的利用受温度的影响 (表 5、表 6)。相关分析表明,猴群对乔木的总体利 用频率与平均温度成正比 $(r=0.664, n=12, P=0.018)$ 。受食时, 猴群对崖壁的利用频率和对裸岩的利用频率 均与平均温度呈负相关关系 (崖壁: $r=-0.685, n=12, P=0.014$; 裸岩: $r=-0.600, n=12, P=0.039$ )。休息时,猴 群对乔木的利用频率与平均温度成正比 $(r=0.650, n=12, P=0.022)$ 。其余的利用频率与平均温度的相关性 没有达到统计学显著水平 (表 5、表 6)。 
表 5 山体部位利用与平均温度的相关性检验结果 $(n=12)$

Table 5 Spearman rank correlations between limestone hill parts used and average temperature $(n=12)$

\begin{tabular}{|c|c|c|c|c|c|}
\hline & & \multicolumn{4}{|c|}{ 山体部位 Hill part } \\
\hline & & 山顶 Hill-top & 崖壁 Cliff & 山坡 Slope & 山脚 Valley \\
\hline \multirow[t]{2}{*}{ 总体 Overall } & $r$ & 0.557 & -0.301 & 0.217 & 0.119 \\
\hline & $P$ & 0.060 & 0.342 & 0.499 & 0.712 \\
\hline \multirow[t]{2}{*}{ 受食 Feeding } & $r$ & 0.263 & -0.685 & 0.448 & 0.127 \\
\hline & $P$ & 0.408 & 0.014 & 0.145 & 0.694 \\
\hline \multirow[t]{2}{*}{ 移动 Moving } & $r$ & -0.229 & 0.046 & -0.028 & - \\
\hline & $P$ & 0.474 & 0.888 & 0.931 & - \\
\hline \multirow[t]{2}{*}{ 休息 Resting } & $r$ & 0.174 & -0.161 & -0.056 & -0.393 \\
\hline & $P$ & 0.588 & 0.618 & 0.863 & 0.206 \\
\hline \multirow[t]{2}{*}{ 社会活动 Social activity } & $r$ & -0.047 & 0.007 & -0.007 & -0.393 \\
\hline & $P$ & 0.885 & 0.983 & 0.983 & 0.206 \\
\hline
\end{tabular}

表 6 微生境利用与平均温度的相关性检验结果 $(n=12)$

Table 6 Spearman rank correlations result between microhabitat used and average temperature $(n=12)$

\begin{tabular}{|c|c|c|c|c|c|}
\hline & & \multicolumn{4}{|c|}{ 微生境类型 Microhabitat } \\
\hline & & 乔木 Tree & 灌木 Bush & 藤本 Liana & 裸岩 Bare rock \\
\hline \multirow[t]{2}{*}{ 总体 Overall } & $r$ & 0.664 & 0.042 & -0.287 & -0.497 \\
\hline & $P$ & 0.018 & 0.897 & 0.366 & 0.101 \\
\hline \multirow[t]{2}{*}{ 受食 Feeding } & $r$ & 0.371 & 0.063 & -0.455 & -0.600 \\
\hline & $P$ & 0.236 & 0.846 & 0.138 & 0.039 \\
\hline \multirow[t]{2}{*}{ 移动 Moving } & $r$ & -0.088 & -0.131 & 0.280 & 0.228 \\
\hline & $P$ & 0.787 & 0.685 & 0.379 & 0.476 \\
\hline \multirow[t]{2}{*}{ 休息 Resting } & $r$ & 0.650 & 0.254 & -0.109 & -0.434 \\
\hline & $P$ & 0.022 & 0.427 & 0.737 & 0.159 \\
\hline \multirow[t]{2}{*}{ 社会活动 Social activity } & $r$ & 0.133 & 0.022 & -0.195 & -0.175 \\
\hline & $P$ & 0.681 & 0.945 & 0.544 & 0.587 \\
\hline
\end{tabular}

\section{3 讨论}

\section{1 食物对栖息地利用的影响}

食物的空间分布对白头叶猴选择栖息地有重要影响。白头叶猴取食的植物在栖息地不同山体部位之间 有变化,因而猴群会选择在山体的不同位置活动 ${ }^{[18]}$ 。山坡是白头叶猴主要的受食场所, 而且猴群受食时,在 食物资源丰富的雨季对山坡的利用频率明显高于食物资源缺乏的旱季,同时相应地减少对崖壁的利用,这与 第一个假设相符。白头叶猴对山体的利用是应对喀斯特石山的地形以及不同季节植物数量、分布存在差异的 一种适应性策略。由于山体各个部位的温度、湿度和土壤质量不同, 植物分布存在明显的空间差异 ${ }^{[2,20,32-33]}$, 随着山体部位往上, 植物种类逐渐减少 ${ }^{[20,32]}$, 即潜在的食物丰富度从平地到山顶逐渐降低。因此, 白头叶猴 选择山坡作为主要的受食场所,且在雨季对该山体部位的利用明显多于旱季,可能与山坡分布着丰富的食物 资源有关。相似地,同域分布的熊猴和猕猴也会根据食物种类的分布而利用山体不同部位 ${ }^{[11]}$ 。

对许多灵长类来说, 捕食风险也可能影响对栖息地的利用, 并且存在受食利益和捕食风险之间的权衡, 选 择既能满足食物需求又能规避风险的山体利用策略, 如黑叶猴 ${ }^{[5]}$ 、熊猴 ${ }^{[4]}$ 和金头叶猴 (Trachypithecus poliocephalus $)^{[34-35]}$ 等。本研究中白头叶猴对山体的选择也表现出同样的策略。白头叶猴更倾向于在崖壁活 动, 崖壁是除受食行为外其他行为的主要活动场所, 而对山顶和山脚的利用很少, 这与第一个假设相符。山脚 分布着丰富的食物资源的同时也面临着更大的捕食风险, 山顶则相反 ${ }^{[2]}$ 。崖壁地势较高, 且视野较开阔, 人 
类及其他陆地动物难以到达, 同时也更容易发现捕食者,有利于规避潜在敌害,因此白头叶猴更多地利用崖 壁。另一方面, 白头叶猴对裸岩的利用主要在山顶和崖壁,而在山坡和山脚多利用更有利于隐藏的乔木, 几乎 不利用裸岩,这也可能说明了捕食风险的重要影响。相似的利用策略还出现在其他灵长类上,例如熊猴多使 用陡峭的山顶和崖壁,这时它们更多利用地面,而猕猴主要使用山坡,可能是为了避开陆地捕食者的攻击因而 倾向待在树上 ${ }^{[9]}$ 。

白头叶猴对微生境的利用模式可能也是基于受食利益和捕食风险相互权衡的结果。从总体、受食、移动 和休息行为来看,白头叶猴主要利用的微生境类型为乔木。一方面,白头叶猴采食的主要植物种类是乔木,如 青檀 (Pteroceltis tatarinowii)、毛叶铁榄(Sinosideroxylon pedunculatum) 和榕属 (Ficus) 植物等乔木为其提供了大 量的食物来源 ${ }^{[36]}$ 。另一方面, 高大的乔木为猴群提供了良好的休息环境和隐蔽场所 ${ }^{[37-38]}$ 。藤本植物和乔木 一样, 既是猴群重要的食物来源又是其隐蔽的重要场所, 而且数量很多 ${ }^{[37]}$, 因此这可能是猴群受食时对藤本 的利用仅次于乔木的原因。猴群进行社会活动时对微生境的利用与其他行为有较大的差异, 如猴群可能会选 择风险相对较低的地方进行理毛 ${ }^{[39]}$, 相似地, 白头叶猴在进行社会活动时更多利用人类及其他陆地动物难以 到达、视野更开阔,更容易提前发现捕食者的崖壁和裸岩, 且对崖壁的利用频率极高,这可能是为了降低捕食 风险作出的选择。

\section{2 温度对栖息地利用的影响}

温度对灵长类利用栖息地有显著影响 ${ }^{[14]}$ 。本研究中, 白头叶猴在移动和休息时选择的微生境主要是乔 木,而且对乔木的利用有明显的季节性差异,休息时对乔木利用的季节性变化尤其明显。猴群在雨季对乔木 的利用明显高于旱季,这与第二个假设相符。猴群对乔木利用的季节性变化可能与行为热调节有关。研究地 点为雨热同季, 雨季(夏季) 时温度较高 ${ }^{[40]}$ 。对于动物来说, 选择荫凉处是应对夏季炎热较为有效的方式, 因 为树林是最有效的遮阴场所, 叶片蒸发水分吸收大量的热量, 因此达到降低空气温度的目的 ${ }^{[41]}$ 。虽然本研究 没有测量林下与非林下的地表温度和裸岩的温度, 但根据已有研究, 研究地点林下的全年平均温度和平均最 高温度明显低于非林下, 尤其是平均最高温度相差近 $15^{\circ} \mathrm{C}^{[24]}$ 。因此, 白头叶猴更多地利用乔木除了可能与受 食利益相关, 与研究地点较高的温度也有密切关系。对微生境利用与温度的相关关系表明, 平均温度越高, 白 头叶猴对乔木的利用频率越高, 对崖壁和裸岩的利用率刚好相反, 这是它们避开高温环境的策略。而在寒冷 的冬季, 动物经常通过主动靠近吸收热量和减少热量散失的方式保持体温 ${ }^{[42]}$ 。本研究也发现白头叶猴在冬 季会选择在更温暖的裸岩表面晒太阳。白头叶猴灵活的行为热调节能力使得其在环境温度的变化下选择利 用不同的栖息地,以达到维持体温恒定的目的。

综上所述, 白头叶猴栖息地利用模式是在受食利益和捕食风险之间作出权衡的结果,并受到温度的影响。 这种栖息地利用模式反映了白头叶猴灵活的行为热调节能力及其对喀斯特石山生境的适应。

\section{参考文献 (References) :}

[ 1 ] 颜忠诚, 陈永林. 动物的生境选择. 生态学杂志, 1998, 17(2): 43-49.

[ 2 ] Li Z Y, Rogers M E. Are limestone hills a refuge or essential habitat for white-headed langurs in Fusui, China? International Journal of Primatology, 2005, 26(2): 437-452.

[ 3 ] 黄乘明, 薛跃规, 韦毅, 李友邦. 白头叶猴栖息环境与栖息地选择的研究. 兽类学报, 2000, 20(3): 180-185.

[ 4 ] 李锋慧, 周岐海, 黄中豪. 广西弄岗熊猴的雨季游走行为与栖息地的利用. 广西师范大学学报: 自然科学版, 2017, 35(4): 114-121.

[ 5 ] 陈婷, 黄中豪, 黄乘明, 周岐海, 韦华. 广西弄岗黑叶猴栖息地选择与利用. 生态学报, 2019, 39(18): 6908-6915.

[ 6 ] Li Y B, Huang X H, Huang Z H. Behavioral adjustments and support use of François' langur in limestone habitat in Fusui, China: implications for behavioral thermoregulation. Ecology and Evolution, 2020, 10(11): 4956-4967.

[ 7 ] McFarland R, Barrett L, Costello M A, Fuller A, Hetem R S, Maloney S K, Mitchell D, Henzi P S. Keeping cool in the heat: behavioral thermoregulation and body temperature patterns in wild vervet monkeys. American Journal of Physical Anthropology, 2020, 171 (3) : 407-418.

[ 8 ] Zhou Q H, Luo B, Wei F W, Huang C M. Habitat use and locomotion of the François' langur (Trachypithecus francoisi) in limestone habitats of Nonggang, China. Integrative Zoology, 2013, 8(4) : 346-355.

[ 9 ] Huang Z H, Huang C M, Wei H, Tang H X, Krzton A, Ma G Z, Zhou Q H. Factors influencing positional behavior and habitat use of sympatric 
macaques in the limestone habitat of Nonggang, China. International Journal of Primatology, 2015, 36( 1): 95-112.

[10] Albani A, Cutini M, Germani L, Riley E P, Ngakan P O, Carosi M. Activity budget, home range, and habitat use of moor macaques ( Macaca maura) in the karst forest of South Sulawesi, Indonesia. Primates, 2020, 61(5): 673-684.

[11] Zhou Q H, Wei H, Tang H X, Huang Z H, Krzton A, Huang C M. Niche separation of sympatric macaques, Macaca assamensis and M. mulatta, in limestone habitats of Nonggang, China. Primates, 2014, 55(1) : 125-137.

[12] Li Z Y, Rogers M E. Food items consumed by white-headed langurs in Fusui, China. International Journal of Primatology, 2006, 27 ( 6) : $1551-1567$.

[13] Zhou Q H, Wei F W, Li M, Huang C M, Luo B. Diet and food choice of Trachypithecus francoisi in the Nonggang Nature Reserve, China. International Journal of Primatology, 2006, 27( 5) : 1441-1460.

[14] Bicca-Marques J C, Calegaro-Marques C. Behavioral thermoregulation in a sexually and developmentally dichromatic neotropical primate, the blackand-gold howling monkey (Alouatta caraya). American Journal of Physical Anthropology, 1998, 106(4) : 533-546.

[15] 谢东明, 路纪琪, 吕九全. 太行山猕猴的冬季生境选择. 兽类学报, 2009, 29(3): 252-258.

[16] 黄中豪, 唐小平, 蒙渊君, 唐华兴, 黄乘明, 周岐海. 黑叶猴在旱季对喀斯特石山生境的利用. 四川动物, 2013, 32(1): 12-18.

[17] Campos F A, Fedigan L M. Behavioral adaptations to heat stress and water scarcity in white-faced capuchins ( Cebus capucinus) in Santa Rosa National Park, Costa Rica. American Journal of Physical Anthropology, 2009, 138(1) : 101-111.

[18] 黄乘明. 中国白头叶猴. 桂林: 广西师范大学出版社, 2002.

[19] IUCN. The IUCN red list of threatened species. (2020-01) [2020-06-30]. http://www.iucnredlist.org.

[20］苏宗明，赵天林，黄庆昌. 弄岗自然保护区植被调查报告. 广西植物，1988，(S1)：185-214.

[21] 黄乘明, 李友邦, 周岐海. 白头叶猴对喀斯特石山生境的适应. 生物学通报, 2016, 51(1): 1-4.

[22] Wang W, Qiao Y, Li S, Pan W, Yao M. Low genetic diversity and strong population structure shaped by anthropogenic habitat fragmentation in a critically endangered primate, Trachypithecus leucocephalus. Heredity, 2017, 118(6) : 542-553.

[23] 魏辅文, 娄治平. 中国野生动物保护研究现状. 中国科学院院刊, 2010, 25(6): 668-673.

[24] 林建忠. 白头叶猴群大小对栖息地利用和家域的影响 [D]. 桂林: 广西师范大学, 2019.

[25] 陆施毅, 鲁长虎, 李友邦. 食物可获得性对白头叶猴 (Trachypithecus leucocephalus) 食物季节性组成的影响. 生态学杂志, 2016, 35 (3): 759- 766 .

[26] Altmann J. Observational study of behavior: sampling methods. Behaviour, 1974, 49(3/4) : 227-267.

[27] Li Y B, Ding P, Huang C M, Jiang P P, Wood C. Dietary response of a group of François' langur Trachypithecus francoisi in a fragmented habitat in the county of Fusui, China: implications for conservation. Wildlife Biology, 2009, 15(2): 137-146.

[28] Huang C M, Wei F W, Li M, Li Y B, Sun R Y. Sleeping cave selection, activity pattern and time budget of white-headed langurs. International Journal of Primatology, 2003, 24(4) : 813-824.

[29] Li Z Y, Rogers E. Habitat quality and activity budgets of white-headed langurs in Fusui, China. International Journal of Primatology, 2004, 25 (1) : 41-54.

[30] 黄乘明, 周岐海, 李友邦, 蔡湘文, 魏辅文. 广西扶绥黑叶猴活动节律和日活动时间分配. 兽类学报, 2006, 26(4): 380-386.

[31] Chen T, Huang Z H, Huang C M, Wei H, Zhou Q H. Positional behaviours of François' langur ( Trachypithecus francoisi) in the limestone forest of Nonggang, Guangxi, South-West China. Folia Primatologica, 2020, 91(3) : 170- 187.

[32］梁畴芬, 梁健英, 刘兰芳, 莫新礼. 弄岗植物区系考察报告. 广西植物, 1985, 5(3)：191-209.

[33] Fan P F, Fei H L, Scott M B, Zhang W, Ma C Y. Habitat and food choice of the critically endangered cao vit gibbon ( Nomascus nasutus) in China: implications for conservation. Biological Conservation, 2011, 144(9): 2247-2254.

[34] Schneider I, Tielen I H M, Rode J, Levelink P, Schrudde D. Behavioral observations and notes on the vertical ranging pattern of the critically endangered Cat Ba langur ( Trachypithecus poliocephalus poliocephalus) in Vietnam. Primate Conservation, 2010, 25 (1): 111-117.

[35] Hendershott R, Rawson B, Behie A. Home range size and habitat use by Cat Ba langurs (Trachypithecus poliocephalus) in a disturbed and fragmented habitat. International Journal of Primatology, 2018, 39(4): 547-566.

[36] Li D Y, Yuan P S, Krzton A, Huang C M, Zhou Q H. Dietary adaptation of white-headed langurs in a fragmented limestone habitat. Mammalia, $2016,80(2): 153-162$.

[37] 黄乘明. 白头叶猴 (Presbytis leucocephalus) 对栖息地选择利用与受食生物学 [D]. 北京: 北京师范大学, 1998.

[38] Adams F V, Teichroeb J A. Microhabitat use in Angolan colobus monkeys (Colobus angolensis ruwenzorii) at Nabugabo, Uganda demonstrates intraspecific variability. International Journal of Primatology, 2020, 41(1) : 24-44.

[39] Cords M. Predator vigilance costs of allogrooming in wild blue monkeys. Behaviour, 1995, 132(7/8) : 559-569.

[40］黄承标, 谭学锋, 农旭能. 白头叶猴栖息地的气候生态环境特征. 四川动物, 2006, 25(4): 866-869.

[41] Armstrong D V. Heat stress interaction with shade and cooling. Journal of Dairy Science, 1994, 77( 7) : 2044-2050.

[42] 尚玉昌. 行为生态学 (十二) : 动物的行为热调节 (1). 生态学杂志, 1986, 5(2): 60-63. 